EPJ Web of Conferences 19, 09004 (2012)

DOI: $10.1051 /$ epjconf/20121909004

(C) Owned by the authors, published by EDP Sciences, 2012

\title{
4MOST - 4-meter Multi-Object Spectroscopic Telescope
}

\author{
R.S. de Jong, C. Chiappini, O. Schnurr and the 4MOST consortium \\ Leibniz Institut für Astrophysik Potsdam (AIP), An der Sternwarte 16, 14482 Potsdam, \\ Germany
}

\begin{abstract}
The 4MOST consortium aims to provide the ESO community with a fibre-fed spectroscopic survey facility on a $4 \mathrm{~m}$-class telescope with a large enough field-of-view (FoV) to survey a large fraction of the southern sky in a few years, a multiplex and spectral resolution high enough to detect chemical and kinematic substructure in the stellar halo, bulge and thin and thick discs of the Milky Way, and enough wavelength coverage ( $>1.5$ octave) to secure velocities of extra-galactic objects over a large range in redshift. 4MOST will run permanently on the selected telescope to perform a 5 year public survey yielding more than 7 million (goal $>25$ million) spectra at resolution $\mathrm{R} \sim 5000$ and more than 1 million spectra at $\mathrm{R} \gtrsim 20,000$. Such an exceptional facility enables many science goals, but our design is especially intended to complement three key all-sky, space-based observatories of prime European interest: Gaia, eROSITA and Euclid.
\end{abstract}

\section{INTRODUCTION}

The 4MOST consortium (AIP, LMU, MPE, and ZAH in Germany, GEPI in France, RAL and IoA in the UK, NOVA and RUG in the Netherlands, and Lund and Uppsala in Sweden) is currently performing a conceptual design study of a wide-field spectroscopic survey facility on either VISTA or NTT. The main facility characteristics are: large FoV of 2 degrees diameter (goal 3 degrees), high fibre multiplex of at least 1500 (goal > 3000), spectrographs of resolution $\mathrm{R} \sim 5000$ covering at least 400-900 nm (goal $390-1050 \mathrm{~nm}$ ) and a spectrograph of $\mathrm{R} \gtrsim 20,000$ covering $390-459$ and $584-676 \mathrm{~nm}$. The instrument is foreseen to be operating permanently on the telescope performing public surveys with catalogs of high-level data products becoming available on yearly. The aim is to start operations early 2018.

\section{SCIENCE DRIVERS}

The Gaia satellite will provide distances from parallaxes and kinematics from proper motions for more than one billion Milky Way stars down to $m_{V} \sim 20$ mag. Gaia will also provide radial velocities and astrophysical characterisation for about 150 million stars, but its sensitivity is limited to $m_{V} \sim 12-$ 16 mag, strongly dependent on stellar spectral type, because its spectrograph only covers the Ca II-triplet region. Figure 1 shows how, by covering the full optical wavelength region, the 4MOST facility complements Gaia, so that full 6D-space coordinate information can be obtained and chemical characterization can be done throughout the Milky Way. Large-area surveys of faint Galactic stellar objects will enable us to elucidate the formation history of the Milky Way.

Models of hierarchical galaxy formation predict large amounts of dynamical substructure in the Milky Way halo that 4MOST can detect through measuring red giant branch (RGB) stars (see Fig. 2). Furthermore, with 4MOST stellar surveys will be used to determine the three-dimensional Galactic

This is an Open Access article distributed under the terms of the Creative Commons Attribution-Noncommercial License 3.0, which permits unrestricted use, distribution, and reproduction in any noncommercial medium, provided the original work is properly cited. 

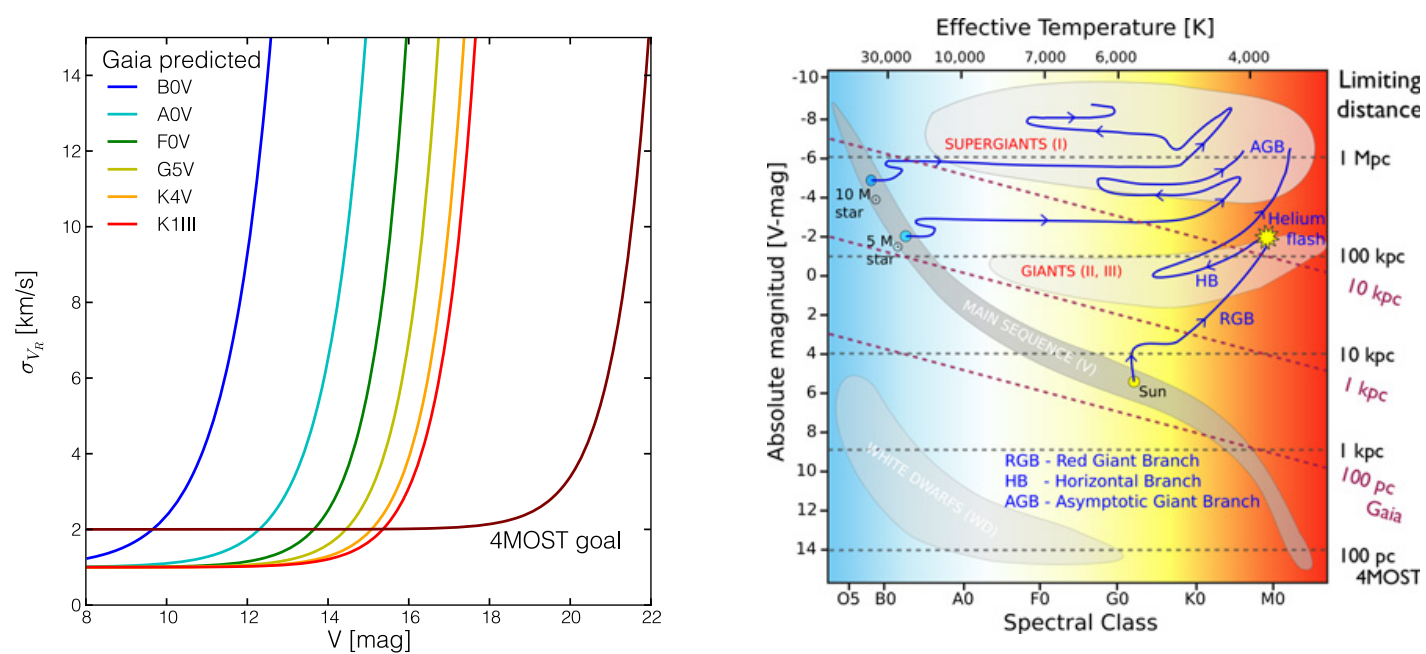

Figure 1. Left: The 4MOST goal for radial velocity accuracy compared to the Gaia end of mission accuracy as function of stellar apparent magnitude. Our aim is to match the spectroscopic magnitude limits of 4MOST to the astrometric limits of Gaia, thereby enabling 6D-phase space studies to Gaia's limits. Right: Limiting distances for radial velocity measurements with Gaia (maroon diagonal) and 4MOST (black horizontal) overlaid on a Hertzsprung-Russell diagram. 4MOST can measure Sun-like stars to nearly the centre of the Milky Way, RGB stars to $100 \mathrm{kpc}$, and massive stars throughout the Local Group, substantially expanding on Gaia's spectroscopic view. Distance limits for the 4MOST high resolution spectroscopy are about four times smaller.

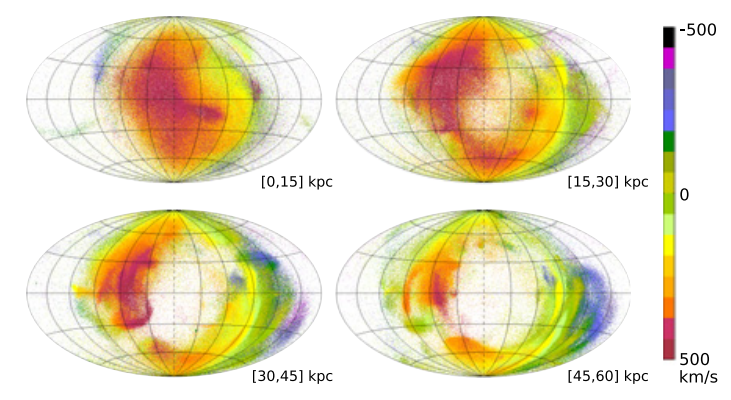

Figure 2. Spatial and radial velocity substructure distribution of RGB stars on the sky for a stellar halo formed in the Aquarius project cosmological simulations $([1,2])$. The projection corresponds to stars located in the inner halo in four distance bins and clearly demonstrates the large amount of substructure that becomes apparent, consequent on the opening of a new phase-space dimension.

potential and its substructure, discern the dynamical structure of the Milky Way disc and measure the influence of its bar and spiral arms, measure the Galactic assembly history through chemo-dynamical substructure and abundance pattern labelling, and find thousands of extremely metal-poor stars to constrain early galaxy formation and the nature of the first stellar generations in the Universe.

Many other science cases are enabled by 4MOST. Its unique capabilities and its operation in public survey mode enable surveys that require 1000s to millions of objects spread over a large fraction of the sky. The goal is to cover a large fraction of the southern sky ( $\gtrsim 20,000$ sq. deg.) twice in a 5 year survey. Of special interest are the followup of the eROSITA X-ray and Euclid dark energy surveys. With 4MOST we will characterise several hundreds of thousands of dynamo- and accretion-powered Galactic X-ray emitters, thereby uncovering the active Milky Way and constraining evolutionary channels of stellar populations. 4MOST will also observe $>50,000$ X-ray detected galaxy clusters, determining 
the evolution of galaxy populations in clusters, yield the cluster mass evolution, and provide highly competitive constraints on dark energy evolution. 4MOST enables us to determine the nature of $>1$ million X-ray detected AGNs, thus constraining the cosmic evolution of active galaxies to $\mathrm{z}=5$. Other science cases that are fully feasible with the 4MOST facility include: constraining dark energy properties through baryon acoustic oscillation (BAO) measurements, the dynamic structure and content of nearby galaxies, and the follow-up of extragalactic radio and infrared surveys.

\section{References}

[1] Cooper, A. P. et al. 2010, MNRAS, 406, 744

[2] Helmi, A. et al. 2011, ApJL, 733, L7 